\title{
Cooling radiation and the Ly alpha luminosity of forming galaxies
}

\section{Citation}

Fardal, Mark A., Neal Katz, Jeffrey P. Gardner, Lars Hernquist, David H. Weinberg, and Romeel Dave. 2001. "Cooling Radiation and the Lya Luminosity of Forming Galaxies." The Astrophysical Journal 562 (2): 605-17. https://doi.org/10.1086/323519.

\section{Permanent link}

http://nrs.harvard.edu/urn-3:HUL.InstRepos:41381819

\section{Terms of Use}

This article was downloaded from Harvard University's DASH repository, and is made available under the terms and conditions applicable to Other Posted Material, as set forth at http:// nrs.harvard.edu/urn-3:HUL.InstRepos:dash.current.terms-of-use\#LAA

\section{Share Your Story}

The Harvard community has made this article openly available.

Please share how this access benefits you. Submit a story.

Accessibility 


\title{
Cooling Radiation and the Lyman-alpha Luminosity of Forming Galaxies
}

\author{
Mark A. Fardal, Neal Katz \\ Astronomy Department, University of Massachusetts, Amherst, MA 01003 \\ Jeffrey P. Gardner \\ Department of Astronomy, University of Washington, Seattle, WA 98195 \\ Lars Hernquist \\ Department of Astronomy, Harvard University, Cambridge, MA 02138 \\ David H. Weinberg \\ Astronomy Department, Ohio State University, Columbus, OH 43210 \\ Romeel Davé \\ Astrophysical Sciences, Princeton University, Princeton, NJ 08544
}

\begin{abstract}
We examine the cooling radiation from forming galaxies in hydrodynamic simulations of the LCDM model (cold dark matter with a cosmological constant), focusing on the Ly $\alpha$ line luminosities of high-redshift systems. Primordial composition gas condenses within dark matter potential wells, forming objects with masses and sizes comparable to the luminous regions of observed galaxies. As expected, the energy radiated in this process is comparable to the gravitational binding energy of the baryons, and the total cooling luminosity of the galaxy population peaks at $z \approx 2$. However, in contrast to the classical picture of gas cooling from the $\sim 10^{6} \mathrm{~K}$ virial temperature of a typical dark matter halo, we find that most of the cooling radiation is emitted by gas with $T<20,000 \mathrm{~K}$. As a consequence, roughly $50 \%$ of this cooling radiation emerges in the Ly $\alpha$ line. While a galaxy's cooling luminosity is usually smaller than the ionizing continuum luminosity of its young stars, the two are comparable in the most massive systems, and the cooling radiation is produced at larger radii, where the Ly $\alpha$ photons are less likely to be extinguished by dust. We suggest, in particular, that cooling radiation could explain the two large $(\sim 100 \mathrm{kpc})$, luminous $\left(L_{\mathrm{Ly} \alpha} \sim 10^{44} \mathrm{erg} \mathrm{s}^{-1}\right)$ "blobs" of Ly $\alpha$ emission found in Steidel et al.'s (1999) narrow band survey of a $z=3$ proto-cluster. Our simulations predict objects of the observed luminosity at about the right space density, and radiative transfer effects can account for the observed sizes and line widths. We discuss observable tests of this hypothesis for the nature of the Ly $\alpha$ blobs, and we present predictions for the contribution of cooling radiation to the Ly $\alpha$ luminosity function of galaxies as a function of redshift.
\end{abstract}


Subject headings: cosmology: theory — galaxies: formation — galaxies: ISM

\section{INTRODUCTION}

Radiative cooling of gas in dark matter potential wells is an essential element of the current theoretical understanding of galaxy formation. As the baryons condense into tightly bound clumps at the centers of these potential wells, they must radiate away the energy that they acquire through compression and shock heating. At first glance, it appears that this radiation must be negligible compared to the radiation produced by stars: the gravitational binding energy per unit mass is $\sim v^{2} \sim 10^{-6} c^{2}$ even for a (high) internal velocity $v \sim 300 \mathrm{~km} \mathrm{~s}^{-1}$, while the nuclear energy per unit mass released in enriching gas to solar metallicity is $\sim 10^{-4} c^{2}$. However, for primordial composition gas cooling to $T \approx 10^{4} \mathrm{~K}$, all of the emitted radiation is either hydrogen or helium line radiation (primarily hydrogen $\mathrm{Ly} \alpha$ ) or continuum radiation above the hydrogen ionization threshold. Only the hottest stars emit photons at these energies. Furthermore, the cooling radiation is produced mainly at large radii, where it can escape the galaxy without being absorbed by dust. Finally, the kinetic energy of supernova explosions is deposited in dense, interstellar gas, where much of it can emerge in the form of hydrogen line emission and ionizing continuum radiation.

Because of these factors, cooling radiation could make a significant contribution to the X-ray, UV, and hydrogen line emission from young galaxies. In this paper, we examine this contribution using hydrodynamic cosmological simulations. Although many such simulations incorporate radiative cooling, most studies have not followed the emitted radiation in detail. One notable exception is the work of Cen \& Ostriker $(1992,1996)$, who calculate the spectrum of the background produced by cooling radiation, but these papers do not examine the emission on a galaxy by galaxy basis. Closer to the focus of this paper is the work of Katz (1992), which examined the formation of a single, massive galaxy, finding that the amount of gravitational energy radiated in atomic lines was comparable to the amount of energy injected by supernovae. Here we examine the cooling radiation from the whole population of forming galaxies, though the individual objects in our simulations are not as well resolved as that of Katz (1992).

The other main approach to theoretical modeling of galaxy formation utilizes semi-analytic methods, in the tradition of White \& Frenk (1991). These methods rely on simplifying assumptions about gravitational collapse and gas dynamics - in particular, they usually assume that infalling gas shock heats to the virial temperature of the dark matter halo, before cooling to join the central object. Our results below suggest that this assumption may break down in the messy assembly process that characterizes hierarchical galaxy formation.

The observational study of star formation in high-redshift galaxies has accelerated in recent years, with progress in wavelength ranges from the UV/optical (Steidel et al. 1996; Williams et al. 1996) to the IR (Elbaz 1999) to the sub-mm (Barger et al. 1999). The present paper is motivated mainly by recent progress in Ly $\alpha$ emission line searches. Historically, this approach to finding high- 
redshift galaxies has proved difficult (Pritchet 1994; Thompson, Djorgovski and Trauger 1995), in part because the $\operatorname{Ly} \alpha$ emission associated with star-forming regions may be damped by the combined effects of resonant scattering and dust absorption. However, Ly $\alpha$ emission line searches have recently begun to bear fruit, thanks to improvements in sensitivity and efficiency (Hu, Cowie \& McMahon 1998; Steidel et al. 1999; Rhoads et al. 2000).

One of the most striking results of these searches is the discovery by Steidel et al. (1999, hereafter S99) of two large regions of diffuse Ly $\alpha$ emission, in their narrow band observations of a galaxy proto-cluster at $z=3.1$. These "blobs" do not resemble the typical galaxies detected in the proto-cluster. They have characteristic angular sizes of 15 " and fluxes of $\approx 1.3 \times 10^{-15} \mathrm{erg} \mathrm{cm}^{-2} \mathrm{~s}^{-1}$. For the cosmological model that we adopt in this paper $\left(\Omega_{m}=0.4, \Omega_{\Lambda}=0.6\right)$, these properties imply proper linear sizes of $l \approx 75 h^{-1} \mathrm{kpc}$ and luminosities of $L_{L y \alpha} \approx 4.4 \times 10^{43} h^{-2} \mathrm{erg} \mathrm{s}^{-1}{ }^{1}$ Some other workers may have observed similar objects. Francis (1999) found diffuse Ly $\alpha$ blobs in a cluster at $z=2.4$. Keel et al. (1999) found three diffuse regions of Ly $\alpha$ emission with sizes of $\sim 40 h^{-1} \mathrm{kpc}$ and luminosities of $\sim 10^{43} h^{-2} \mathrm{erg} \mathrm{s}^{-1}$ (in our adopted cosmology), also at $z=2.4$. These are somewhat smaller and less luminous than the blobs found by S99, and some are located around known AGN, so they may or may not be related phenomena.

Several explanations for these Ly $\alpha$ blobs have been proposed. They could be caused by photoionization from a hidden central AGN (S99). High-redshift radio galaxies often show emission line regions with sizes and luminosities comparable to the S99 blobs, and the absence of radio lobes could simply indicate a radio-quiet AGN; the anisotropic emission of the standard unified AGN model would then provide an explanation for the absence of nuclear optical emission. Another possibility, also discussed in S99, is that the gas in the blobs is photoionized by ultraviolet (UV) continuum radiation from young stars. The required star formation rate is $\sim 30 f_{\text {esc }}^{-1} M_{\odot} \mathrm{yr}^{-1}$, where $f_{\text {esc }}$ is the fraction of hydrogen ionizing radiation that escapes from the galaxy. Low-redshift observations imply upper limits of $f_{\text {esc }} \lesssim 10 \%$ (Leitherer et al. 1995; Hurwitz, Jelinsky \& Dixon 1997 ), so unless these presumably gas-rich galaxies have much larger $f_{\text {esc }}$, the required star formation rates are substantial. However, current limits are quite weak, allowing rates $\lesssim 1000 M_{\odot} \mathrm{yr}^{-1}$ (Steidel et al. 1999). Taniguchi \& Shioya (2000) have suggested that the Ly $\alpha$ emission emerges from a galactic superwind that is powered by high star formation rates and extends to scales that are even larger than those of winds observed at low redshift (Heckman, Lehnert, \& Armus 1993). While the luminosities and sizes of these blobs can be explained by wind models, the mechanism for channeling the wind energy into Ly $\alpha$ emission is somewhat unclear.

Drawing on the results of our more general investigation, we propose an alternative explanation for the Ly $\alpha$ blobs: they represent gas that is radiating away its gravitational potential energy as it settles into massive galaxies. We describe our numerical simulation techniques, with particular attention to our treatment of radiative cooling, in $\S 2$. In $\S 3$ we present our results for the cooling radiation and Ly $\alpha$ emission from forming galaxies. In $\S 4$ we discuss whether the cooling radiation

\footnotetext{
${ }^{1}$ With $\Omega_{m}=1, \Omega_{\Lambda}=0$, these numbers become $l \approx 55 h^{-1} \mathrm{kpc}$ and $L_{L y \alpha} \approx 2.4 \times 10^{43} h^{-2} \mathrm{erg} \mathrm{s}^{-1}$.
} 
model can account for the observed properties of the Ly $\alpha$ blobs. Section 5 discusses some of the numerical uncertainties in our calculations, compares our model to a similar model proposed independently by Haiman, Spaans, \& Quataert (2000) using semi-analytic methods, summarizes our results and discusses their implications and predictions.

\section{SIMULATIONS}

We perform our simulations using the parallel version of the cosmological N-body/hydrodynamic code TreeSPH (Hernquist \& Katz 1989; Katz, Weinberg, \& Hernquist 1996a; Davé, Dubinski, \& Hernquist 1997), a code that unites smoothed particle hydrodynamics (SPH) (Lucy 1977; Gingold \& Monaghan 1977) with the hierarchical tree method for computing gravitational forces (Barnes \& Hut 1986; Hernquist 1987). Dark matter, stars, and gas are all represented by particles; collisionless material is influenced only by gravity, while gas is subject to gravitational forces, pressure gradients, and shocks. The gas can also cool both radiatively, assuming primordial abundances, and through Compton cooling.

TreeSPH is fully adaptive in both space and time. In SPH, gas properties are computed by averaging or "smoothing" over a fixed number of neighboring particles, 32 in the calculations here. Hence the smoothing lengths in TreeSPH decrease in collapsing regions, in proportion to the interparticle separation, and in underdense regions the smoothing lengths are larger. TreeSPH allows particles to have individual time steps according to their physical state, so that the pace of the overall computation is not driven by the small fraction of particles requiring the smallest time steps. There is a maximum allowed timestep, called the system timestep, and all particles are integrated with this step or one a power of two smaller. The timestep criteria are detailed further in Katz, Weinberg, \& Hernquist (1996a) and Quinn et al. (2000); we set the tolerance parameter $\eta$ to 0.4 .

Since this paper concerns cooling radiation, it is important to understand how we evolve the thermal energy. Usually one has two choices: to integrate the thermal energy equation explicitly or implicitly. Integrating explicitly would require a timestep about three times smaller than the cooling time, which is much smaller than what would be required to integrate the dynamical equations, making the calculation prohibitively expensive computationally. To integrate the equations implicitly involves inverting an $N_{\text {gas }} \times N_{\text {gas }}$ matrix several times per timestep. With $N_{\text {gas }}>3 \times 10^{6}$ in our simulations, this would also be prohibitively expensive. Instead we take an intermediate approach, solving the thermal energy equation semi-implicitly as described in Hernquist and Katz (1989). Briefly, we integrate the changes in the thermal energy caused by shocks and pressure forces explicitly, while we integrate those caused by radiative cooling implicitly. Since the radiative cooling depends only on a particle's own temperature and density, independent of other particles, no matrix inversion is required, and the time scale of the non-radiative processes is comparable to the other time scales that govern the gas timestep. 
The semi-implicit approach guarantees that we integrate the thermal energy equation in a stable way, but it does not guarantee accuracy. Accuracy is maintained by keeping the size of the timestep reasonably small. We accomplish this in two ways. First, we integrate the thermal energy equation for all gas particles, independent of their dynamical timestep, using a timestep that is one half the size of the smallest dynamical timestep of any particle. Second, we damp the cooling rate so that no gas particle loses more than a given fraction of its thermal energy in one timestep (see Katz \& Gunn 1991); i.e., we slightly change the physics to make the numerical integration more robust. This could have the effect of making some regions temporarily hotter than they would have been if these numerical compromises were not made. In practice, it just makes some regions take two or three timesteps to cool instead of one, since these regions have such short cooling times. This could change the temperature at which the energy is radiated, but it should not change the total radiated energy by a large amount.

The simulations calculate radiative cooling processes assuming a primordial composition gas and ionization equilibrium. These processes include collisional excitation, radiative and dielectronic recombination, collisional ionization, and bremsstrahlung. Molecular processes and metal lines are omitted, which means that radiative cooling alone cannot reduce the temperature below $\sim 10^{4} \mathrm{~K}$. The gas cooling rates we use for these processes are listed in Katz, Weinberg, \& Hernquist (1996a, see their figure 1). However, the rate at which the gas radiates energy is somewhat different from the rate at which it loses thermal energy: the emitted recombination radiation includes both the actual gas cooling and the atomic ionization potential, while collisional ionization removes thermal energy but yields no radiation at all. A possible deficiency of the simulations is the omission of He I line cooling, but we have verified that it is unimportant compared to the other processes in the simulations presented here.

For the purposes of this paper, we are particularly interested in the Ly $\alpha$ emission. We assume that a fraction 0.68 of recombinations to H I produce a Ly $\alpha$ photon, appropriate for optically thick gas at $10^{4} \mathrm{~K}$; accounting for the relative photon energies this channels 0.49 of the recombination energy through Ly $\alpha$. In practice this is usually a small contribution compared to the collisional excitation. Excitation of $\mathrm{H}$ I by collisions with electrons can result in either a Ly $\alpha$ photon or in 2-photon decay from the metastable $2{ }^{2} \mathrm{~S}$ state, as well as photons from higher series in some cases. The ratio between Ly $\alpha$ and 2-photon decay is weakly dependent on temperature. By averaging over one simulation, we find that a fraction 0.59 of the $\mathrm{H}$ I collisional excitation is channeled through Ly $\alpha$ with almost all of the remaining energy in the 2-photon continuum.

TreeSPH can also include a metagalactic ionizing UV background field. TreeSPH does not include radiative transfer, so even dense regions, which in reality should be self-shielded, are exposed to the full background field. This results in a large energy exchange and unphysical gas radiation from these regions. Hence, we have restricted our analysis to simulations performed without such a background, which should yield reliable results for our purposes.

In these simulations we include star formation and supernova feedback using the algorithm 
described in Katz, Weinberg, \& Hernquist (1996a). The algorithm forms stars in dense cool regions at a rate essentially controlled by the dynamical supply of gas. For each star formation event, supernova energy is added to the surrounding particles as thermal energy on a time scale of $2 \times 10^{7}$ years.

In the version of our code used for these simulations, the thermal energy input from supernovae is added at the beginning of each system timestep, not continuously. The system timesteps are always larger than the cooling time in these dense star forming groups, so the excess thermal energy has radiated away by the end of the step when we output the system state. This actually turns out to be quite helpful, since the remaining radiation is caused by the radiating away of gravitational energy and thus can be measured in isolation. We can easily recover the supernova thermal input because it is proportional to the star formation rate. Using a Miller-Scalo initial mass function (IMF) with cutoffs at 0.1 and $100 M_{\odot}$, the heat input from supernovae is $2.7 \times 10^{41} \mathrm{erg} \mathrm{s}^{-1} M_{\odot}^{-1} \mathrm{yr}$ when smoothed over the neighboring particles (the exact value would be $2.5 \times 10^{41} \mathrm{erg} \mathrm{s}^{-1} M_{\odot}^{-1} \mathrm{yr}$ if there were no smoothing). The drawback to this method is that we cannot easily compute the distribution of the supernova energy within galaxies or the processes by which it is radiated away. Since the supernova energy is deposited in the dense ISM over a length scale determined by our resolution, its space and temperature distributions are somewhat suspect in any case, and we will not attempt to reconstruct them.

In addition to cooling radiation, we compute the photoionizing radiation from hot stars from the simulation outputs. Using the code STARBURST99 (Leitherer et al. 1999), we find a Lyman continuum intensity of $2.6 \times 10^{42} \mathrm{erg} \mathrm{s}^{-1} M_{\odot}^{-1} \mathrm{yr}$ for a Miller-Scalo IMF, assuming a mean Lyman continuum photon energy of 1.4 Ryd. For gas at $10^{4} \mathrm{~K}$, there are 0.68 Ly $\alpha$ photons emitted per photoionization (Charlot \& Fall 1993), giving an associated Ly $\alpha$ emissivity of $9.4 \times 10^{41} \mathrm{erg} \mathrm{s}^{-1} M_{\odot}^{-1} \mathrm{yr}$. Because the stars that produce photoionizing radiation are short-lived, we can take the stellar emissivity to be proportional to the instantaneous star formation rate, which we compute from the gas distribution. We do not include this UV emission or stellar winds as a source of feedback in the simulations. It is likely to have even less dynamical impact than supernova feedback, since any energy captured by the gas is deposited in the densest, star forming regions with moderate temperatures, where it can be quickly radiated away.

To summarize, the three sources of radiative energy included in these simulations that could result in Ly $\alpha$ radiation are gravitational cooling, supernova feedback, and photoionizing input from hot stars. These sources have distinct physical origins, our code follows them separately, and we will keep them separate in the discussions and figures. When we refer to the "total cooling", we mean the gravitational cooling summed over all radiative processes, not that we are including the supernova or photoionizing input.

We calculate the cooling radiation at discrete output times with the analysis program TIPSY, ${ }^{2}$

\footnotetext{
${ }^{2}$ http://www-hpcc.astro.washington.edu/TSEGA/tools/tipsy/tipsy.html
} 
using the same cooling algorithm as TreeSPH. To identify discrete objects in the simulations, we use the program SKID. ${ }^{3}$ Because SKID defines gravitationally bound groups of particles, we will refer to these objects as "groups," though each such group actually represents a single galaxy. SKID slides particles meeting density and temperature criteria along density gradients until they reach a local maximum. We use a density cutoff corresponding to the edge of a virialized isothermal halo, or a gas density of $\rho_{g}=\left(\Omega_{b} / \Omega_{m}\right)\left(\rho_{v i r} / 3\right)$, where the mean density of a spherical virialized halo $\rho_{v i r}$ is 178 at early times and is given in general by Kitayama \& Suto (1996). As in Weinberg et al. (1999), we also restrict ourselves to groups that contain more than 64 baryonic particles, roughly the mass resolution limit of the simulations. Once we identify the groups, we can easily add up the cooling radiation for all particles in the group. Cooling radiation can also be emitted outside these groups, but it proves to be a minor contribution except at very early times; most of this additional emission comes from groups excluded by our mass resolution criterion rather than from low density gas.

All three of the simulations we discuss in this paper assume a $\Lambda$-dominated cold dark matter cosmological model with $\Omega_{m}=0.4, \Omega_{\Lambda}=0.6, h \equiv H_{0} /\left(100 \mathrm{~km} \mathrm{~s}^{-1} \mathrm{Mpc}^{-1}\right)=0.65$, and a primeval spectral index $n=0.93$. With the tensor mode contribution, normalizing to COBE using CMBFAST (Seljak \& Zaldarriaga 1996; Zaldarriaga, Seljak, \& Bertschinger 1998), implies a normalization $\sigma_{8}=0.8$, which provides a good match to cluster abundances (White, Efstathiou, \& Frenk 1993). We use the Hu \& Sugiyama (1996, equation D28) formulation of the transfer function. We adopt a baryonic density $\Omega_{b}=0.02 h^{-2}$ consistent with the deuterium abundance in high redshift Lyman limit systems (Burles \& Tytler 1997, 1998) and with the opacity of the Ly $\alpha$ forest (Rauch et al. 1997). All of our simulations model a triply periodic cubical volume.

The main and largest simulation we discuss is the L144 simulation (Davé et al. 2000), with $144^{3}$ gas and dark matter particles, a box length of $50 \mathrm{~h}^{-1}$ comoving Mpc on a side, and a gravitational softening length $\epsilon_{\text {grav }}=7 \mathrm{~h}^{-1}$ comoving kpc (equivalent Plummer softening). The nominal gas mass resolution is $5.4 \times 10^{10} M_{\odot}$, corresponding to 64 gas particles. To investigate the effects of our finite resolution we perform two additional simulations. The L11/64 simulation has a higher spatial and mass resolution, so it must be run in a smaller volume, $11.1 h^{-1}$ comoving Mpc on a side. It has $64^{3}$ gas and dark matter particles, $\epsilon_{\text {grav }}=3.5 \mathrm{~h}^{-1}$ comoving kpc, and a gas mass resolution of $6.8 \times 10^{9} M_{\odot}$. The L11/32 simulation is identical to the L11/64 simulation, with the same initial phases, except that it uses $32^{3}$ particles of each type and has the same resolution as the L144 simulation. In all the simulations the nominal spatial resolution in physical units is $\sim 2 \epsilon_{\text {grav }} /(1+z)$.

\footnotetext{
${ }^{3}$ http://www-hpcc.astro.washington.edu/TSEGA/tools/skid.html
} 


\section{COOLING RADIATION AND THE LY $\alpha$ LUMINOSITY FUNCTION}

Figure 1 displays the temperature distribution of the total gravitational cooling radiation (all radiative processes), in the $\mathrm{L} 144$ simulation at $z=3$ and $z=0$. In the conventional theoretical sketch of galaxy formation (e.g., White \& Rees 1978; White \& Frenk 1991), gas that falls into dark matter halos is shock heated to the virial temperature $T_{\text {vir }}=\left(\mu m_{p} / 2 k\right) v_{c}^{2} \approx 10^{6} \mathrm{~K}\left(v_{c} / 165 \mathrm{~km} \mathrm{~s}^{-1}\right)^{2}$, then cools and settles into the central galaxy. The dotted line in Figure 1 shows the temperature distribution of cooling radiation expected for gas cooling from an initial temperature of $10^{6} \mathrm{~K}$. The numerical simulation results paint a very different picture. A large fraction of the cooling radiation, $75 \%$ at $z=3$ and $30 \%$ at $z=0$, comes from gas with $10^{4} \mathrm{~K}<T<2 \times 10^{4} \mathrm{~K}$. Most of the remaining radiation comes from much hotter gas, with $10^{6} \mathrm{~K}<T<10^{8} \mathrm{~K}$.

The lack of cooling radiation from gas with $2 \times 10^{4} \mathrm{~K}<T<10^{6} \mathrm{~K}$ cannot be a result of gas "moving quickly" across this temperature range, since even if it did, it would still have to radiate away its thermal energy in order to reach $2 \times 10^{4} \mathrm{~K}$. Instead, Figure 1 implies that most of the gas that cools into galaxies is never heated to the virial temperature of a galaxy-mass dark halo. This conclusion accords with that of Kay et al. (2000), who find, based on similar sorts of simulations, that only $\sim 10 \%$ of SPH particles that end up in galaxies were ever heated above $10^{5} \mathrm{~K}$. We find qualitatively similar results in our own simulations, but we have not examined particle temperature trajectories with the high (single timestep) time resolution used by Kay et al. (2000).

This physical result has major implications for the spectrum of cooling radiation from forming galaxies, since much of the energy from neutral gas at $T \sim 10^{4} \mathrm{~K}$ emerges in the Ly $\alpha$ line as a result of collisional excitation (see Katz, Weinberg, \& Hernquist 1996a, figure 1). The hot gas cooling, on the other hand, is dominated by bremsstrahlung. At $z=3$, Ly $\alpha$ emission accounts for $43 \%$ of the cooling radiation in the simulation, with $29 \%$ emerging in HI 2-photon emission, $19 \%$ in bremsstrahlung, and only $8 \%$ in other radiative processes. If we assumed a metal-enriched intergalactic medium instead of primordial composition, then more of the hot gas might be able to cool, boosting the fraction of radiation from $10^{5} \mathrm{~K}<T<10^{7} \mathrm{~K}$, but the large emissivity of lower temperature gas would remain.

Our focus in this paper is the cooling radiation, and especially the $\operatorname{Ly} \alpha$ emission, associated with individual galaxies. In Figure 2, the dot-dashed line shows the cumulative luminosity function of the total gravitational cooling at $z=3$. By "total" we mean that this includes all of the cooling radiation processes, not just Ly $\alpha$ radiation. The reradiated supernova energy is plotted as the dotted line. The gravitational energy available to a group increases more quickly with mass than the star formation rate. The dominant source of the total cooling radiation thus changes over from reradiated supernova energy to gravitational energy as the mass and luminosity increase. The two processes combined give the luminosity function shown by the solid line. Finally, the dashed line shows the stellar UV emissivity, calculated from the star formation rate in the galaxy. For all luminosities, there is more energy in the UV radiation produced by the massive stars than in the cooling radiation. However, the UV radiation from young stars is likely to be heavily absorbed by 
dust, and reradiated in the infrared. The gravitational cooling radiation emerges from lower density gas and is more likely to escape. The supernova energy may also stand a much better chance of escaping, as supernovae can destroy the dense clouds responsible for the heaviest absorption and deposit their kinetic energy in a more diffuse medium.

We plot the cumulative luminosity function of the Ly $\alpha$ cooling radiation alone in Figure 3, at several redshifts. Since the fraction of supernova energy converted into Ly $\alpha$ is uncertain, we include only gravitational sources of cooling in this plot. Figure 2 shows that gravitational cooling dominates in the most luminous objects. The number of highly luminous objects reaches a peak at $z=2$, and declines thereafter. At $z=3$ there are $\sim 4 \times 10^{-5} h^{3} \mathrm{Mpc}^{-3}$ objects in the simulation with Ly $\alpha$ luminosities greater than $3 \times 10^{43} h^{-2} \mathrm{erg} \mathrm{s}^{-1}$, comparable to the "blobs" of S99.

In the left hand panel of Figure 4, we show a map of the Ly $\alpha$ emission contributed by gravitational energy in one of our simulated groups, and in the right hand panel we plot the stellar photoionization using a conversion factor of star formation to recombination-induced Ly $\alpha$ of $6.6 \times 10^{41} \mathrm{erg} \mathrm{s}^{-1} M_{\odot}^{-1} \mathrm{yr}$. The emission is shown by the gray scale images, with the intensity scale marked in terms of $\log _{10}\left[I_{L y \alpha} /\left(\operatorname{erg~s}^{-1} \mathrm{~cm}^{-2} \operatorname{asec}^{-2}\right)\right]$. The emission from gravitational cooling is more spatially extended than the stellar emission. Emission of cooling radiation from reradiated supernova feedback would look like a scaled version of the right hand panel, slightly smoothed by the feedback algorithm. We also plot contours of the neutral hydrogen column density, calculated assuming an ionizing background of $3 \times 10^{22} \mathrm{erg} \mathrm{s}^{-1} \mathrm{~cm}^{-2} \mathrm{~Hz}^{-1} \mathrm{sr}^{-1}$ and correcting for self-shielding (Katz et al. 1996b). Self-shielding accounts for the rather sharp edges seen in the neutral gas (cf. Maloney 1993). These emission plots ignore radiative transfer effects and dust extinction, both of which could greatly alter the observed appearance of these systems. We discuss these issues in $\S 4$.

The spatial extent of the emission for the groups in the L144 simulation is shown in Figure 5. The measure we use here is the root mean square distance from the center of the group, weighted by the Ly $\alpha$ emissivity. The dotted line shows the gravitational softening length; recall that the nominal spatial resolution is about twice this length. We plot the Ly $\alpha$ extent against the rms extent of the gas mass defined in a similar manner. We also show the size weighted by star formation rate, which would represent the size of the stellar UV emissivity region. In all cases, the star formation in our simulations occurs in a small, partially resolved region at the center of the group. The Ly $\alpha$ emission usually emerges over a region comparable to the size of the gas as a whole and is well resolved numerically. However, the most luminous groups in the simulation tend to have more concentrated emission; although there is weak emission at large radii, the typical rms sizes are less than $10 \mathrm{kpc}$. This size is much smaller than that of the blobs observed by S99, a point we will return to in $\S 4$.

We plot the gravitational cooling in $\operatorname{Ly} \alpha$ as a function of galactic mass in Figure 6. We define the galactic mass to be the stellar mass plus the mass of the gas that is at least 1000 times overdense and has temperature $T<3 \times 10^{4} \mathrm{~K}$. To increase our dynamic range, we show both the L144 simulation and the higher resolution (but smaller volume) L11/64 simulation. There 
is a strong correlation between the emitted Ly $\alpha$ cooling radiation and the galactic mass. In the L144 simulation, the galaxies with high Ly $\alpha$ cooling luminosities are all high mass objects, many corresponding to $L_{*}$ galaxies or above.

In Figure 7 we plot the total gravitational cooling radiation as a function of the star formation rate at $z=3$. Supernova feedback would add an amount of cooling radiation shown by the dashed line. The Lyman continuum luminosity associated with the stellar UV emission is marked along the top axis. Star formation rates are underestimated in marginally resolved systems, a numerical artifact that causes the spread in these scatter plots towards low star formation rates at relatively low cooling luminosity. Figure 7 demonstrates on an object-by-object basis the features seen in the luminosity functions of Figure 2. Supernova cooling dominates gravitational cooling in low mass objects, but gravitational cooling takes over at high masses. The objects with the highest cooling luminosity also have high star formation rates $\left(\mathrm{SFR} \gtrsim 100 M_{\odot} \mathrm{yr}^{-1}\right.$ ). The stellar UV luminosity always exceeds the gravitational cooling radiation (all points representing well resolved objects lie below the dotted line), but they are of similar magnitude in the most luminous objects, so the cooling radiation would dominate if the stellar UV is heavily extinguished by dust.

To examine the energetics of the cooling radiation, we would like to define the gravitational potential energy available to the baryons in the groups, but this definition is quite ambiguous. Rather than being symmetric, isolated entities, the groups are embedded in a complex, filamentary, and clumpy structure. We are interested in the energy available to the baryons, but they interact with the dark matter in a complex manner and energy is transferred between them. Nevertheless, it is interesting to see whether the potential energy, with the matter outside the group ignored, is a good predictor of the cooling radiation.

In all of the previous figures, the groups defined by SKID consist of baryonic particles only. To study the gravitational energy, we must redefine the groups to include dark matter particles as well, using a density cutoff of $\rho_{t o t}=\rho_{v i r} / 3$. If we defined $U_{t o t}$ to be the total potential energy of the group, and if the baryons and dark matter were distributed identically with a baryon fraction $f_{b}$, and if we assigned half of the baryon-dark matter interaction energy to the baryon potential energy $U_{b}$, then we would have $U_{b}=f_{b} U_{t o t}$. Another possibility would be to assign the entire interaction energy to the baryons. For example, if the cooling and collapse of baryons to the center of the group takes place when the dark matter halo is already assembled, then this latter definition is closer to the amount of energy that the gas radiates. As a practical matter, $M_{b} V_{c}^{2}$, where $V_{c}$ is the circular velocity and $M_{b}$ is the baryonic mass, usually lies between these two definitions. Hence, we take $M_{b} V_{c}^{2}$ as our estimate of the potential energy of the baryons. One might object that, by the virial theorem, the energy available is only $\left|U_{b}\right| / 2$. However, the virial theorem does not apply to the baryons alone, as they are confined by the potential of the dark matter. ${ }^{4}$ As a rule the sum of the kinetic and thermal energies falls short of even the smaller definition of $\left|U_{b}\right|$ by a factor of 2

\footnotetext{
${ }^{4}$ In addition, the group as a whole is not an isolated system, and in our simulations the gravitational force is softened and thus not a power law; both of these facts also violate the conditions for the virial theorem to hold.
} 
to 4 .

A simple estimate of the cooling radiation is then $M_{b} V_{c}^{2} / t$, the potential energy divided by the cosmic time. At $z=3$ this prescription somewhat underestimates the cooling from each group, as shown in Figure 8, though the discrepancy is not large considering the ambiguity in our definition of $\left|U_{b}\right|$. At $z=0$, this simple formula overestimates the cooling, indicating that the cooling occurs preferentially at early times. There could be several reasons for this trend. The cooling could be taking place less effectively since the virial temperatures are rising; the rate of accretion of matter into the group could have slowed; or the baryons could become more effective at transferring their energy to the dark halos. We are unable to determine the answer from the discrete outputs of the current simulations. Tracking the evolution of distinct groups as a function of time, as was done by Katz (1992) for a single galaxy, would give more insight into the energetics, but we leave this investigation for future work.

\section{MODELING THE LY $\alpha$ BLOBS}

So far, we have established that galaxy-like groups in our simulations generically show large amounts of cooling in Ly $\alpha$. Can this cooling explain the "blobs" observed by S99?

The Ly $\alpha$ luminosities of some of our groups are as large as those of the S99 blobs. The number density of groups in our simulations with a Ly $\alpha$ luminosity of $3 \times 10^{43} h^{-2} \mathrm{erg} \mathrm{s}^{-1}$ is about $4 \times 10^{-5} h^{3} \mathrm{Mpc}^{-3}$. At present we can make only crude estimates of the number density of the observed Ly $\alpha$ blobs. The volume examined by S99 has a size of $8^{\prime} .7 \times 8^{\prime} .9$ and a depth of $\Delta z=0.066$, or a comoving volume of $4200 h^{-3} \mathrm{Mpc}^{3}$ in our adopted cosmology. As long as the blobs are less abundant in other regions of space, they have a comoving density of $<5 \times 10^{-4} h^{3} \mathrm{Mpc}^{-3}$. A better estimate can be obtained from S99's observation that this region is overabundant in both Lyman break galaxies and in Ly $\alpha$-emitting galaxies by a factor of $\sim 6$. If the blobs are biased in the same way, they have a density of $\sim 8 \times 10^{-5} h^{3} \mathrm{Mpc}^{-3}$, twice the density we find. If these blobs are associated with very massive objects, they are probably more highly biased than Lyman break galaxies; hence their actual number density is probably lower than this estimate. With only two objects, which are probably correlated, the statistical uncertainties are large. So while the current constraints are quite weak, the number density is consistent with that found in our simulations.

The most luminous objects in our simulations typically have high mass and are strongly clustered. For example, the 100 most massive objects in the L144 simulation have a comoving correlation length of $5.7 \mathrm{Mpc}$. This strong clustering is consistent with the discovery of the blobs in a proto-cluster, though of course the abundance of blobs in blind fields is not well known.

As shown in Figures 4 and 5, the length scale over which most of the Ly $\alpha$ cooling is emitted in our simulations is usually much smaller than the observed sizes of the blobs in S99, though it is also larger than the typical size of the star forming regions. Hence, we must appeal to resonant scattering to transport the $\operatorname{Ly} \alpha$ photons to large radii. 
The radiative transfer of Ly $\alpha$ in a static slab is a classic problem (Adams 1972, 1975; Neufeld 1990). The behavior of Ly $\alpha$ photons depends upon the optical depth of the slab. For intermediate optical depths, the Ly $\alpha$ photons are spatially trapped, and they escape by scattering into the tails of the Doppler distribution, where the slab is optically thin. For very large optical depths, the photons escape by scattering into the damping wings, where they then perform a random walk in both space and frequency. The transition between these two regimes occurs roughly where the damping wings become optically thick. For gas with a temperature $T_{4} \equiv T /\left(10^{4} \mathrm{~K}\right)$, the total central optical depth is $\tau_{0}=\left(N_{H I} / 1.7 \times 10^{13} \mathrm{~cm}^{-2}\right) T_{4}^{-1 / 2}$, and the damping constant is $a=4.7 \times 10^{-4} T_{4}^{-1 / 2}$. The damping wings, which have the line profile $\phi(x)=a /\left(\pi x^{2}\right)$, become optically thick at $\tau_{0} \approx 4 \times 10^{4}$, or $N_{H I} \approx 7 \times 10^{17} T_{4}^{1 / 2} \mathrm{~cm}^{-2} .{ }^{5}$

Despite the extensive work on the slab problem, we have not found calculations of the typical line-center optical depth at last scattering $\left(\tau_{\text {last }}\right)$ in the literature. We can estimate $\tau_{\text {last }}$ in two regimes. In the case of intermediate optical depth, the spatial diffusion is negligible and $\tau_{\text {last }} \approx \tau_{0} / 2$, the optical depth to the slab center. The photons escape with a double-peaked profile with a typical frequency shift $x \equiv \Delta \nu / \Delta \nu_{D o p}=\sqrt{\ln \tau_{0}}$. For large optical depths, the random excursion that leads to escape takes place at a frequency $x_{*} \sim\left(a \tau_{0} / 2 \sqrt{\pi}\right)^{1 / 3}$ (Adams 1972), for which the optical depth through the slab is $\tau_{*}=\tau_{0} \phi\left(x_{*}\right) \sim\left(a \tau_{0} / 2 \sqrt{\pi}\right)^{1 / 3}$. The last scattering of the photons in that excursion occurs at optical depth $\sim 1$ at that frequency, or a line-center optical depth of $\tau_{\text {last }} \sim \phi\left(x_{*}\right)^{-1} \sim\left(\tau_{0} / 2\right)\left(a \tau_{0} / 2 \sqrt{\pi}\right)^{-1 / 3}$. Without performing Monte Carlo calculations, it is difficult to say exactly where the transition between these regimes occurs; we will take it to be $\tau_{0} \approx 10^{5}$. For very large columns, the Ly $\alpha$ photons are extinguished by dust. This occurs for $N_{H I} \gtrsim 4 \times 10^{20} \mathrm{~cm}^{-2} T_{4}^{1 / 2} \xi_{\text {dust }}^{-3 / 4}$ where $\xi_{\text {dust }}$ is the dust-to-gas ratio relative to the Galactic value (Neufeld 1990). Even though galaxies at high redshift might have low metallicity, star-forming regions should still be fairly rich in dust. We somewhat arbitrarily take $\xi_{d u s t}=0.1$ as a typical value.

Most of the neutral gas in our baryonic groups is at about $10^{4} \mathrm{~K}$. Hence, we might expect that Ly $\alpha$ photons emitted at $N \gtrsim 2 \times 10^{21} \mathrm{~cm}^{-2}$ are absorbed by dust. Photons emitted at $2 \times 10^{18} \mathrm{~cm}^{-2} \lesssim N_{H I} \lesssim 2 \times 10^{21} \mathrm{~cm}^{-2}$ are scattered in the line wings and finally escape in the range $7 \times 10^{17} \mathrm{~cm}^{-2} \lesssim N_{H I} \lesssim 7 \times 10^{19} \mathrm{~cm}^{-2}$. Finally, photons emitted at $N_{H I} \lesssim 2 \times 10^{18} \mathrm{~cm}^{-2}$ are scattered mostly in place and eventually escape not far from their region of formation.

Although we are using one-dimensional models for these estimates, they should be at least crudely applicable to our three-dimensional groups. Applying this picture to Figure 4, we see that Ly $\alpha$ photons originating from stellar photoionization are likely to be absorbed. This is consistent with the strong absorption seen in star-forming galaxies at high redshifts (Smail, Ivison \& Blain 1997). However, many of the photons from gravitational cooling may be scattered outwards to the $10^{18} \mathrm{~cm}^{-2}$ neutral hydrogen contour or about $\sim 30 h^{-1} \mathrm{kpc}$, with velocity widths up to $300 \mathrm{~km} \mathrm{~s}^{-1}$.

\footnotetext{
${ }^{5}$ Note that this column density is much less than what is conventionally referred to as a "damped" line, $N_{H I} \sim$ $10^{20} \mathrm{~cm}^{-2}$.
} 
In general we find that much of the gravitational cooling is emitted outside the region of intense star formation. Usually $\sim 20 \%$ is generated outside our nominal dust cutoff, but this fraction can be smaller or larger depending on the value we choose for the cutoff. A crucial ingredient in this argument is the presence of reservoirs of neutral gas at large radii around the largest groups in our simulations. The velocities obtained are not quite as high as in Figure 8 of S99, but the greatest velocity spread there comes from isolated knots and the velocity of the truly diffuse emission is mostly unconstrained.

Clumping of the gas could make the escape of the photons easier and shrink the apparent size of the Ly $\alpha$-emitting regions (Neufeld 1991). The presence of large bulk motions can greatly affect the line transfer, probably explaining the moderate fraction of cases where photons escape from star-forming regions (Kunth et al. 1998; Ahn \& Lee 1998). However, calculations using the velocity fields of our groups, which are not well resolved in any case, are beyond the scope of this paper. We can conclude at this point only that our model may be consistent with the observed sizes and velocity widths of the Ly $\alpha$ blobs.

The cooling gas model for the blobs makes several testable predictions. Since the Ly $\alpha$ emission is expected to be scattered to larger radii, emission in other lines should be more centrally concentrated. The neutral hydrogen column should be $\sim 10^{18} \mathrm{~cm}^{-2}$ out to the radius of the Ly $\alpha$ blobs. Since the Ly $\alpha$ is caused mostly by collisional excitation rather than photoionization, the $\mathrm{H} \alpha$ flux should be quite small relative to Ly $\alpha(\lesssim 2 \%)$. These predictions are at odds with the results of Francis et al. (1996) and Francis, Woodgate \& Danks (1997), who report a detection of $\mathrm{H} \alpha$ and $\mathrm{CIV}$ in their three blobs and He II Balmer- $\alpha$ in two. In their brightest blob, labeled B1, H $\alpha$ has a similar strength and distribution to Ly $\alpha$. This suggests that at least these blobs are not due to gravitational cooling; this is concordant with the apparent double-lobed morphology of B1 and red stellar colors of the central galaxies, which suggest a AGN origin for these blobs. H $\alpha$ unfortunately falls in the K-band only in the range $2.0<z<2.6$, so it is important to search for other possible lines in the S99 blobs.

Another implication of our model is that large luminosities in diffuse Ly $\alpha$ emission are associated with massive objects with large star formation rates. In fact, if Figure 7 is taken at face value, the star formation rates implied for the S99 blobs are $\sim 100 M_{\odot} \mathrm{yr}^{-1}$. However, the tight relation between cooling luminosity and star formation rate is due to a similarly tight relation between baryonic mass and star formation rate. These quantities may not be as correlated in real galaxies. The more fundamental prediction is that there is a massive galaxy at the heart of each Ly $\alpha$ blob.

As mentioned before, to power the Ly $\alpha$ nebulosity with Lyman continuum radiation from young stars, one needs $\sim 300\left(f_{\text {esc }} / 0.1\right)^{-1} M_{\odot} \mathrm{yr}^{-1}$ where $f_{\text {esc }}$ is poorly known. In the supernova wind model of Taniguchi \& Shioya (2000), the required star formation rates are $\sim 200 M_{\odot} \mathrm{yr}^{-1}$. This rough agreement between the required star formation rates of three different models is somewhat frustrating, with only the model of AGN photoionization allowing a different rate. 


\section{DISCUSSION}

The most basic, and perhaps the most surprising result of this work, is that most of the gravitational cooling radiation comes from gas at $T<2 \times 10^{4} \mathrm{~K}$, far below the typical virial temperatures of galaxies (see Figure 1). In these simulations (and those of Kay et al. 2000), most of the gas that settles into galaxies is never heated to the virial temperature of a galaxy-mass dark matter halo. In $\S 5.1$ we discuss some of the numerical issues surrounding this result. Astronomical implications are discussed in $§ 5.2$.

\subsection{Numerical Uncertainties}

While numerical simulations are a useful guide to physical intuition, they are far from a perfect model of reality. With regard to our principal result - the temperature distribution of the coolingit is easy to think of some limitations of the current code that might affect cooling estimates. For example, the omission of molecules and metals might have some effect. The main effect of molecular cooling would simply be to allow cooling below $10^{4} \mathrm{~K}$ in the densest regions, so it would not affect our Ly $\alpha$ predictions. Metals might channel some of the Ly $\alpha$ cooling into other lines, and it would allow more high temperature gas to cool. However, the metallicity must be above a few percent of solar before it significantly affects the cooling curve, and such a high metallicity seems somewhat unlikely for gas that is falling into galaxies for the first time at high redshift. The key feature that leads to high Ly $\alpha$ luminosity is that most gas that enters high-redshift galaxies is never heated to high $\left(T>10^{5} \mathrm{~K}\right)$ temperatures at all. In this regard, a more worrisome concern is inadequate resolution of shocks, which could allow gas temperatures to rise more slowly than they should.

Concern about numerical resolution in general can be addressed by examining the simulations L11/32 and L11/64. As we mentioned earlier, L11/32 has the same resolution as our main simulation L144, but it covers a smaller volume. L11/64 resimulates the same volume as L11/32 with the same initial density field, but with eight times the mass resolution and twice the spatial resolution. These two resolutions are compared in Figure 9, where we plot the luminosity of the cooling radiation emitted in Ly $\alpha$ for both simulations. Since the initial density fields are identical, we can compare the Ly $\alpha$ cooling luminosity galaxy by galaxy. We find that although the individual Ly $\alpha$ cooling luminosities can vary, the ratio of $\operatorname{Ly} \alpha$ cooling luminosity in the two simulations scatters around one, giving us confidence in our ability to predict the Ly $\alpha$ cooling luminosity in the L144 simulation. The amount of scatter is not surprising in view of the highly stochastic nature we find for the cooling luminosity in individual simulated galaxies.

Though it is encouraging that the cooling luminosity function is consistent between our two different resolutions, the cooling could be altered by resolution effects if the important scales are below the resolution of even the L11/64 simulation. This caveat may undermine our prediction that Ly $\alpha$ should dominate $\mathrm{H} \alpha$ and X-ray emission, as the presence of stronger shocks would increase collisional ionization and recombination radiation. We will eventually be able to test for resolution 
effects over a wider dynamic range, but this will require other, more computationally demanding simulations.

Our method for time integration of the thermal energy also deserves comment. As mentioned above, we damp the cooling when the cooling times are shorter than the minimum timestep. It would be surprising if this substantially affects the total radiated energy. However, if there is an induced error, it is in the direction of causing us to slightly overestimate the temperature of the gas and hence slightly overestimate the importance of X-ray emission relative to Ly $\alpha$ emission.

\subsection{Observational Implications}

Laying aside our numerical concerns, which can only be fully addressed by future work, let us summarize our principal astronomical results. We find that large amounts of gravitationally induced Ly $\alpha$ radiation should be produced around massive galaxies in the early universe. The gravitational cooling radiation is smaller than the stellar UV output, but its relative significance increases with the galaxy mass, and its physical extent is quite different, making it less likely to be extinguished by dust. The Ly $\alpha$ luminosity function extends up to $\sim 10^{44} \mathrm{erg} \mathrm{s}^{-1}$, and it reaches a peak at $z \approx 2$.

We suggest that this gravitational cooling radiation could explain the blobs found by S99. The luminosities, number densities, and clustering are consistent with current constraints. The cooling radiation is dominated by collisional excitation, so that the expected ratio of $\mathrm{H} \alpha$ to Ly $\alpha$ is small. The size of the Ly $\alpha$ emission region is unlikely to be as large as the observed blobs, but we find that sizes approaching those observed can be produced by resonant scattering of the Ly $\alpha$ radiation.

If our model of the blobs is correct, it follows that the currently observed blobs are merely among the brightest members of their class at $z=3$. Our derived luminosity function goes roughly as $d \mathcal{N}(>L) / d L \propto L^{-1.5}$ at S99's luminosity threshold. If the sky background dominates the pixel noise, detecting much fainter objects at $z=3$ will be difficult. However, our luminosity evolution suggests the surface brightnesses of the blobs may peak at $z \sim 1$. Even at $z=2$, we expect $\sim 6$ times as many blobs at S99's limiting surface brightness as at $z=3$ (these numbers are sensitive to cosmology). Ground-based observations at the lowest achievable redshift, $z \approx 2$, may be the optimal method for finding these blobs until the launch of NGST.

The results we find have applications beyond the Ly $\alpha$ blobs. For example, semi-analytic models of galaxy formation generally assume that gas in galaxies should be cooling principally at the virial temperature of the galaxy, and hence be emitted in X-rays. In the picture of White \& Frenk (1991), the gas in a halo shock heats to the virial temperature, then condenses onto the central galaxy from the inside out as it cools. The rate of cooling is given by the minimum of the dynamical gas infall rate and the growth of the mass within the cooling radius, defined to be the radius where the cooling time equals the cosmic time. The emitted cooling is taken to have a near isothermal spectrum at the virial temperature. 
A problem with this picture is that the expected X-ray emission from individual galaxies has not been detected with ROSAT (Benson et al. 1999). One reason for this failure may be that the cooling radiation is not emitted primarily at the virial temperature but instead covers a large range of temperatures, with $10^{4} \mathrm{~K}$ predominant. This in turn suggests that the assembly of gas to form galaxies is a more gentle process than in the White \& Frenk (1991) picture. Once gas has collected into cool dense objects, it seems that it is difficult to force it out of that state, and dissipation mostly takes place through efficient atomic lines. Dominance of low temperature cooling does not necessarily require that galaxy assembly proceed by mergers of discrete, cold objects. If the gas encounters a series of weak shocks as it falls into a galaxy instead of a single strong shock, it may be able to radiate its internal energy as quickly as it acquires it, never reaching high temperature.

During preparation of our paper, a preprint by Haiman et al. (2000) appeared proposing a very similar model for the S99 blobs. We note some of the similarities and differences of the two models here. Haiman et al. (2000) base their analysis on a semi-analytic calculation, using a merger tree formalism and a spherical collapse model. In contrast to previous semi-analytic calculations that assume the energy emerges at the virial temperature, Haiman et al. (2000) argue that cooling will be so efficient that most energy will be radiated at $\sim 10^{4} \mathrm{~K}$. In general, their picture is in reasonable agreement with ours, but it differs in quantitative details. We find lower amounts of Ly $\alpha$ radiation by a factor 2-4, partly because they ignore H I 2-photon emission and bremsstrahlung as coolants. Their treatment of radiative transfer seems to overemphasize the frequency shift due to resonant scattering and underestimate the spatial scattering. Because they assume the Ly $\alpha$ radiation is produced out to the virial radius, they do not consider this a problem. Our emission is somewhat more centrally concentrated. They assume monolithic collapse, so that for a given galaxy there is first a cooling stage and then a star formation stage. In contrast, we find that cooling and star formation happen simultaneously in massive galaxies.

Finally, Haiman et al. (2000) find that the efficiency of star formation in producing Ly $\alpha$ radiation is only twice that due to gravitational cooling. In our simulation this ratio is about 20. The difference comes in part from their channeling all the cooling radiation through Ly $\alpha$ and in part from their assumption about the importance of feedback. They assume that only $10 \%$ of the gas forms stars. Since at the present day most galaxies are dominated by stars rather than gas, this implies the remaining gas must be blown out again by galactic winds. In our simulations, the efficiency of forming stars out of eligible gas is only $10 \%$ at each timestep, but the gas is not blown out, and eventually most of the cooled gas is turned into stars. The effectiveness of feedback in removing gas from galaxies is currently a major question in astrophysics, and the comparison of cooling radiation and stellar emission may eventually help to constrain the answer.

Further theoretical work is needed to test the robustness of our predictions over a wider dynamic range of numerical parameters. Further observational work is needed to test whether the predicted Ly $\alpha$ emission from young galaxies exists in the real universe. This emission, perhaps already observed in the form of the Ly $\alpha$ blobs, represents a novel form of radiation from galaxies that potentially offers a direct view of the process of galaxy formation. 
We thank Chuck Steidel, Martin Weinberg, Chigurupati Murali, Paul Francis, and Mark Voit for helpful discussions. This work was supported by NASA Astrophysical Theory Grants NAG53922, NAG5-3820, and NAG5-3111, by NASA Long-Term Space Astrophysics Grant NAG5-3525,

and by the NSF under grants ASC93-18185, ACI96-19019, and AST-9802568. The simulations were performed at the San Diego Supercomputer Center, NCSA, and the NASA/Ames Research Center.

\section{REFERENCES}

Adams, T. F. 1972, ApJ, 174, 439

Adams, T. F. 1975, ApJ, 201, 350

Ahn, S.-H., \& Lee, H.-W., 1998, astro-ph/9801031

Barger, A. J., Cowie, L. L., Smail, I., Ivison, R. J., Blain, A. W. \& Kneib, J.-P. 1999, AJ, 117, 2656

Barnes, J.E., \& Hut, P. 1986, Nature, 324, 446

Benson, A. J., Bower, R. G., Frenk, C. S., \& White, S. D. M. 1999, astro-ph/9903179

Burles, S., \& Tytler, D. 1997, AJ, 114, 1330

Burles, S., \& Tytler, D. 1998, ApJ, 499, 699

Cen, R., \& Ostriker, J. P. 1992, ApJ, 393, 22

Ostriker, J. P. 1996, \& Cen, R., ApJ, 464, 27

Charlot, S., \& Fall, S. M. 1993, ApJ, 415, 580

Davé, R., Dubinski, J., \& Hernquist, L. 1997, New Astron, 2, 227

Davé, R., Katz, N., Hernquist, L., \& Weinberg, D.H. 2000, in preparation.

Elbaz, D. 1999, astro-ph/9911050

Francis, P. J., Woodgate, B. E., Warren, S. J., Moller, P., Mazzolini, M., Bunker, A. J., Lowenthal, J. D., Williams, T. B., Minezaki, T., Kobayashi, Y., Yoshii, Y. 1996, ApJ, 457, 490

Francis, P. J., Woodgate, B. E. \& Danks, A. C. 1997, ApJ, 482, L25

Francis, P. 1999, talk at Puebla conference

Gingold, R.A. \& Monaghan, J.J. 1977, MNRAS, 181, 375 
Haiman, Z., Spaans, M. \& Quataert, E. 2000, astro-ph/0003366

Heckman, T. M., Lehnert, M. D., \& Armus, L. 1993, The Environment and Evolution of Galaxies, J. M. Shull and H. A. Thronson, Kordrecht: Kluwer, 455

Hernquist, L. 1987, ApJS, 64, 715

Hernquist, L. \& Katz, N. 1989, ApJS, 70, 419

Hu, W. \& Sugiyama, N. 1996, ApJ, 471, 542

Hu, E. M., Cowie, L. L. \& McMahon, R. G. 1998, ApJ, 502, L99

Hurwitz, M., Jelinsky, P. \& Dixon, W. V. D. 1997, ApJ, 481, L31

Katz, N. \& Gunn, J. E. 1991, ApJ, 377, 365

Katz, N. 1992, ApJ, 391, 502

Katz, N., Weinberg D.H., \& Hernquist, L. 1996a, ApJS, 105, 19

Katz, N., Weinberg D.H., Hernquist, L., \& Miralda-Escudé, J. 1996b, ApJ, 457, L57

Kay, S. T., Pearce, F. R., Jenkins, A., Frenk, C. S., White, S. D. M., Thomas, P. A., \& Couchman, H. M. P. 2000, MNRAS, in press, astro-ph/9908107

Keel, W. C., Cohen, S. H., Windhorst, R. A. \& Waddington, I. 1999, AJ, 118, 2547

Kitayama, T., \& Suto, Y. 1996, ApJ, 469, 480

Kunth, D. , Mas-Hesse, J. M., Terlevich, E., Terlevich, R., Lequeux, J. \& Fall, S. M. 1998, A\&A, 334,11

Leitherer, C. , Ferguson, H. C., Heckman, T. M. \& Lowenthal, J. D. 1995, ApJ, 454, L19

Leitherer, C. , Schaerer, D., Goldader, J. D., González Delgado, R. M., Robert, C., Kune, D. F., de Mello, D. F., Devost, D., \& Heckman, T. M. 1999, ApJS, 123, 3

Lowenthal, J. D., Hogan, C. J., Green R. F., Caulet, A., Woodgate, B. E., Brown, L. \& Foltz C. B. 1991, ApJ 377, 73L

Lucy, L. 1977, AJ, 82, 1013

Maloney, P. 1993, ApJ, 414, 41

Neufeld, D. 1990, ApJ, 350, 216

Neufeld, D. 1991, ApJ, 370, L85

Pritchet, C. J. 1994, PASP, 106, 1052 
Quinn, T., Katz, N., Stadel, J. \& Lake, G. 2000, astro-ph/9710043

Rhoads, J. E., Malhotra, S., Dey, A., Stern, D., Spinrad, H., Jannuzi, B. 2000, ApJ, submitted, astro-ph/0003465

Rauch, M., Miralda-Escudé, J., Sargent, W. L. W., Barlow, T. A., Weinberg, D. H., Hernquist, L., Katz, N., Cen, R., \& Ostriker, J. P., 1997, ApJ, 489, 7

Seljak, U., \& Zaldarriaga, M. 1996, ApJ, 469, 437

Smail, I., Ivison, R. J. \& Blain, A. W. 1997, ApJ, 490, L5

Steidel, C. C., Giavalisco, M., Pettini, M., Dickinson, M., \& Adelberger, K. L. 1996, ApJ, 462, L17

Steidel, C. C., Adelberger, K. L., Shapley, A. E., Pettini, M., Dickinson, M., \& Giavalisco, 1999, astro-ph/9910144

Taniguchi, Y. \& Shioya, Y. 2000, ApJ, 532, L13

Thompson, D., Djorgovski, S. and Trauger, J. 1995, AJ, 110, 963

White, S. D. M., Efstathiou, G. P., \& Frenk, C. S. 1993, MNRAS, 262, 1023

White, S. D. M., \& Frenk, C. S. 1991, ApJ, 379, 52

White, S. D. M., \& Rees, M. J. 1978, MNRAS, 183, 341

Weinberg, D. H., Davé, R., Gardner, J. P., Hernquist, L., \& Katz, N. 1999, astro-ph/9908133 (contribution to the Proceedings of Rencontres Internationales de l'IGRAP, Clustering at High Redshift, Marseille 1999)

Williams, R. E., et al. 1996, AJ, 112, 1335

Zaldarriaga, M., Seljak, U., \& Bertschinger, E. 1998, ApJ, 494, 491 


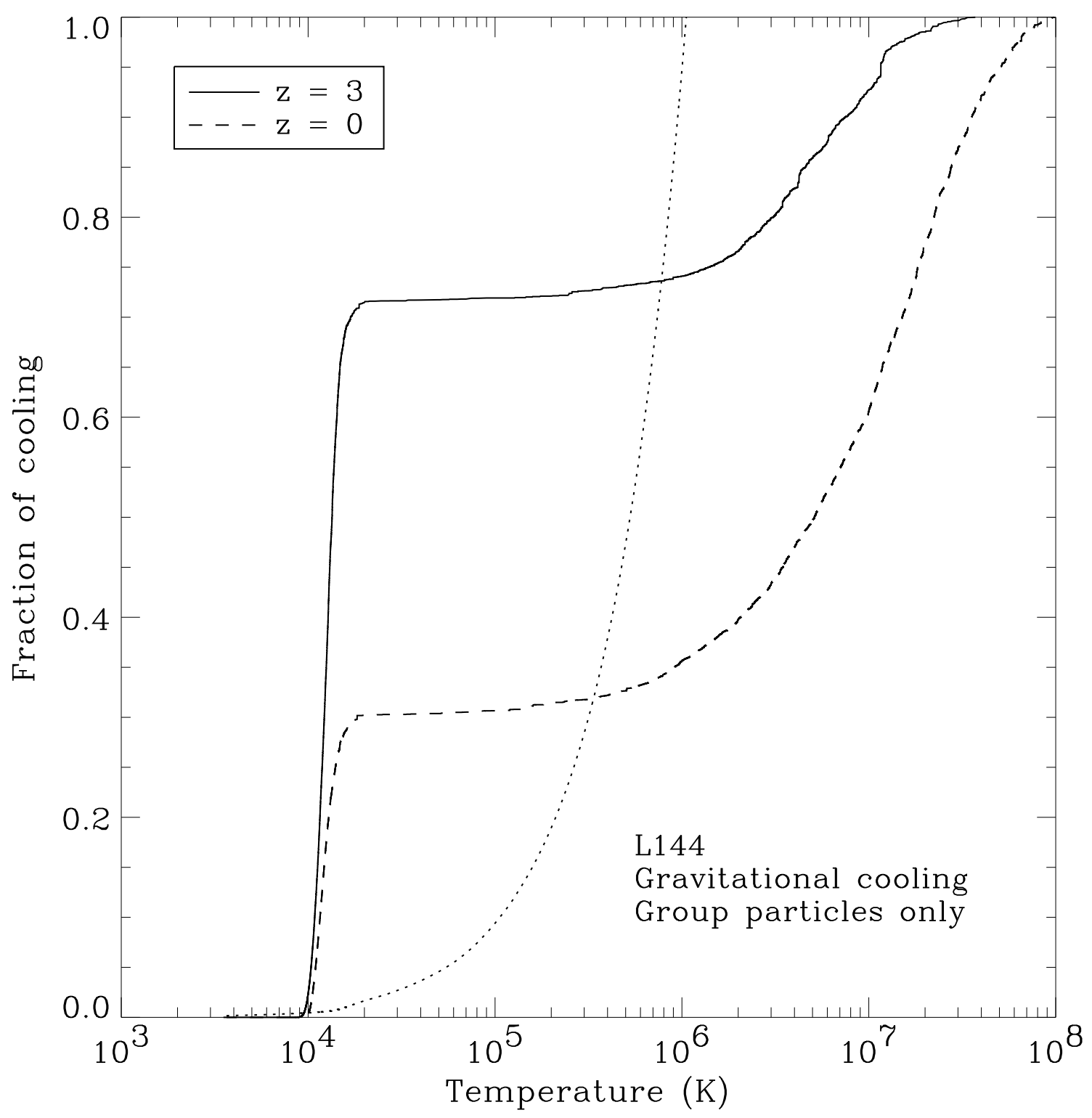

Fig. 1.- Cumulative distribution of the cooling radiation as a function of the temperature of the emitting gas, for the L144 simulation at $z=3$ (solid) and $z=0$ (dashed). The supernova contributions to the cooling are omitted. The dotted line shows the distribution expected for gas that cools from an initial temperature of $10^{6} \mathrm{~K}$. Since the gas is fully ionized in $\mathrm{H}$ and He down to $\sim 8 \times 10^{4} \mathrm{~K}$, this curve is approximately $T /\left(10^{6} \mathrm{~K}\right)$ over most of its range of significance. 


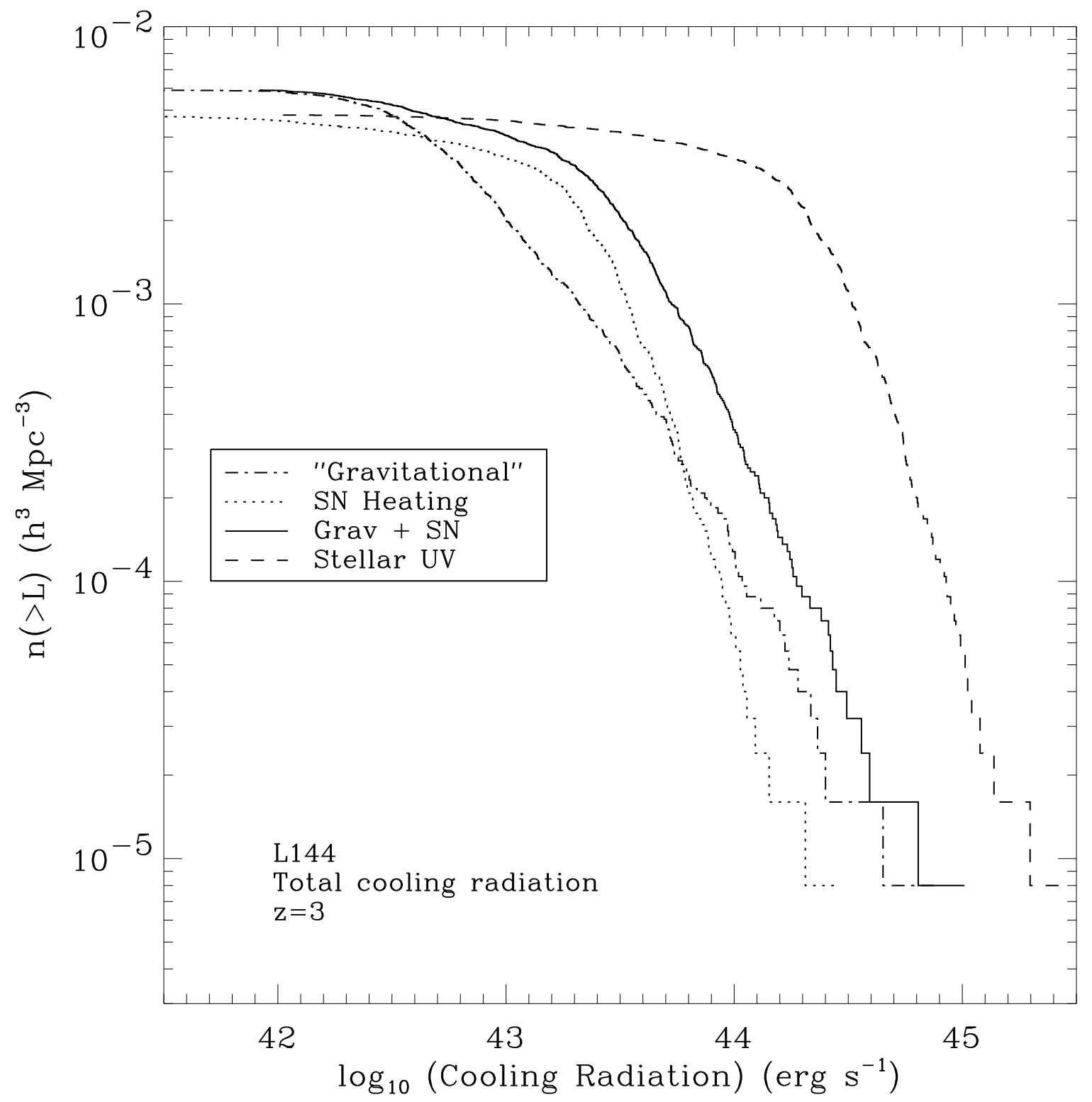

Fig. 2.- Luminosity functions based on the different sources of energy input to the gas. The gravitational cooling luminosity function of groups in the L144 simulation at $z=3$ is plotted as the dot-dashed line. The contributions from supernova heating and stellar Lyman continuum are plotted as the dotted and dashed lines respectively. The sum of gravitational and supernova inputs are plotted as the solid curve. The turnover in the curves at a density of $3 \times 10^{-3} h^{3} \mathrm{Mpc}^{-3}$ is an artifact of limited resolution. All luminosity functions in this paper are plotted using comoving densities. 


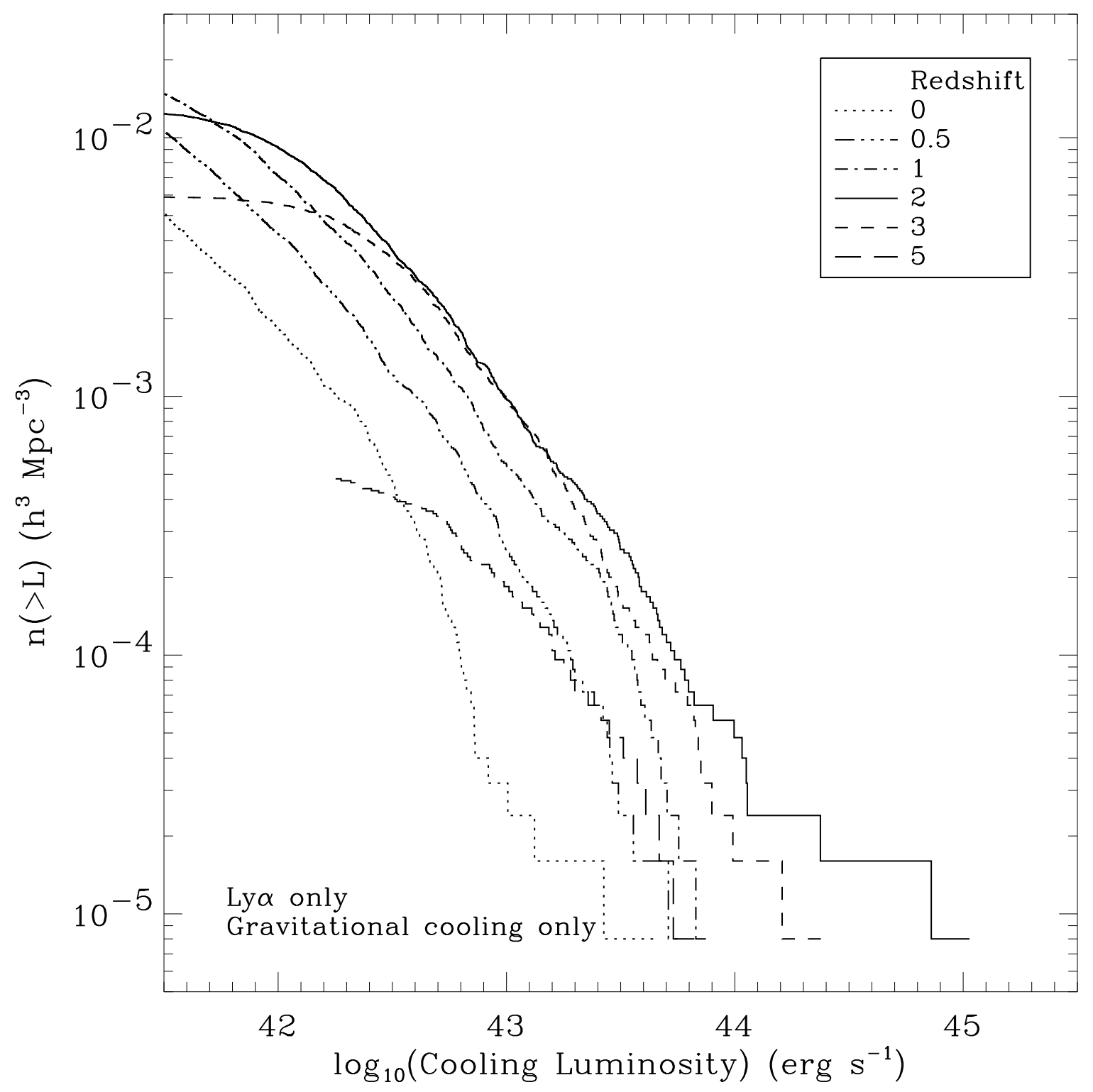

Fig. 3.- Evolution of the cumulative Ly $\alpha$ cooling luminosity function at several redshifts, for the L144 simulation. Only the contribution from gravitational sources is included. 

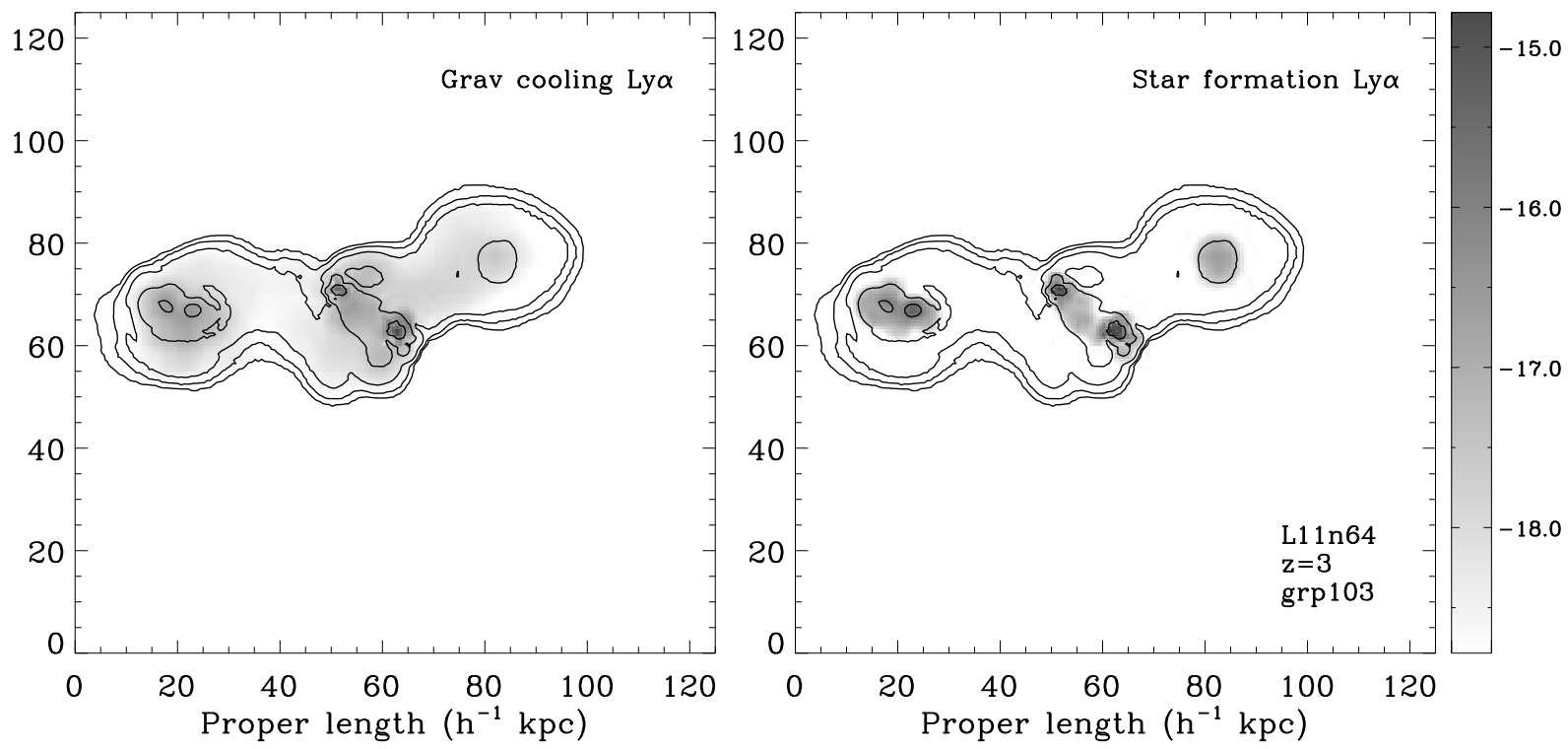

Fig. 4.- An example of the Ly $\alpha$ radiation resulting from gravitational cooling (left panel) and stellar photoionization (right panel) in one of our groups at $z=3$. The example here is taken from our highest resolution simulation L11/64. The emission is shown by the gray scale images; the intensity scale is marked in terms of $\log _{10}\left[I_{L y \alpha} /\left(\operatorname{erg~s}^{-1} \mathrm{~cm}^{-2} \operatorname{asec}^{-2}\right)\right]$. The neutral hydrogen column density is also shown at contours of $\log _{10}\left(N_{\mathrm{HI}} / \mathrm{cm}^{-2}\right)=18,19,20,21$, and 22 . 


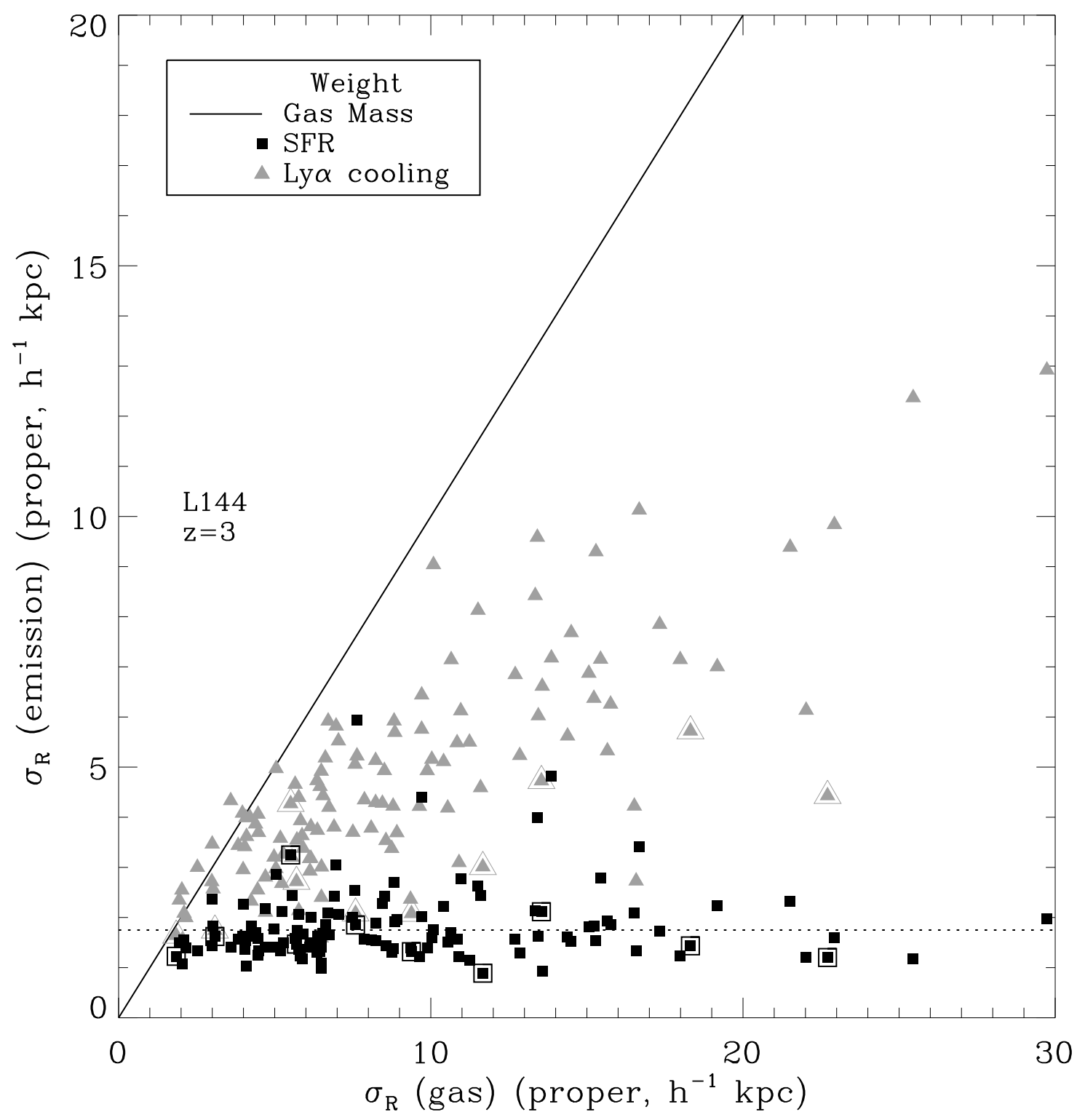

Fig. 5. - Radial rms extent of the gas in the cooling groups at $z=3$, weighted by several different factors. The $x$-axis is weighted by the gas mass. On the $y$-axis, the triangles are weighted by the Ly $\alpha$ emission (considering only the "gravitational" cooling). The squares are weighted by the star formation rate, which is proportional to the stellar ionizing emission. The dotted line shows the gravitational softening length. The points would lie on the solid line if the emissivity were proportional to the gas density. Only groups with Ly $\alpha$ cooling of more than $10^{43} \mathrm{erg} \mathrm{s}^{-1}$ are shown; groups with Ly $\alpha$ cooling of more than $5 \times 10^{43} \mathrm{erg} \mathrm{s}^{-1}$ are shown surrounded by outlines. 


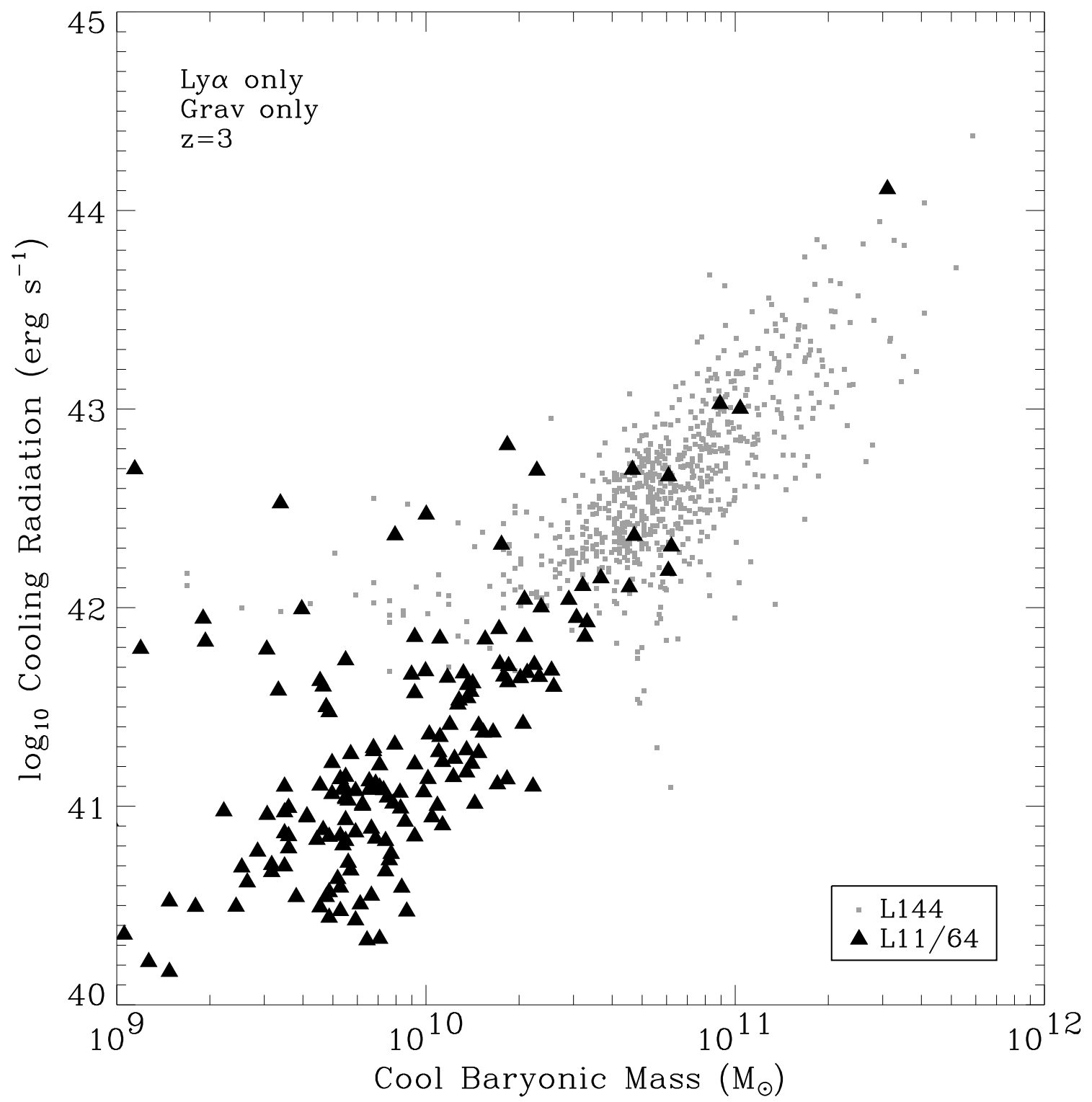

Fig. 6.- Cooling as a function of the "galactic mass", at $z=3$. This is defined as the mass of the gas with temperature $T<3 \times 10^{4} \mathrm{~K}$ and overdensity $\rho_{g} / \overline{\rho_{g}}>1000$, plus the mass in stars. The cooling includes only the gravitational Ly $\alpha$ cooling. The galaxies in the L144 simulation and the L11/64 simulation are plotted as squares and triangles respectively. 


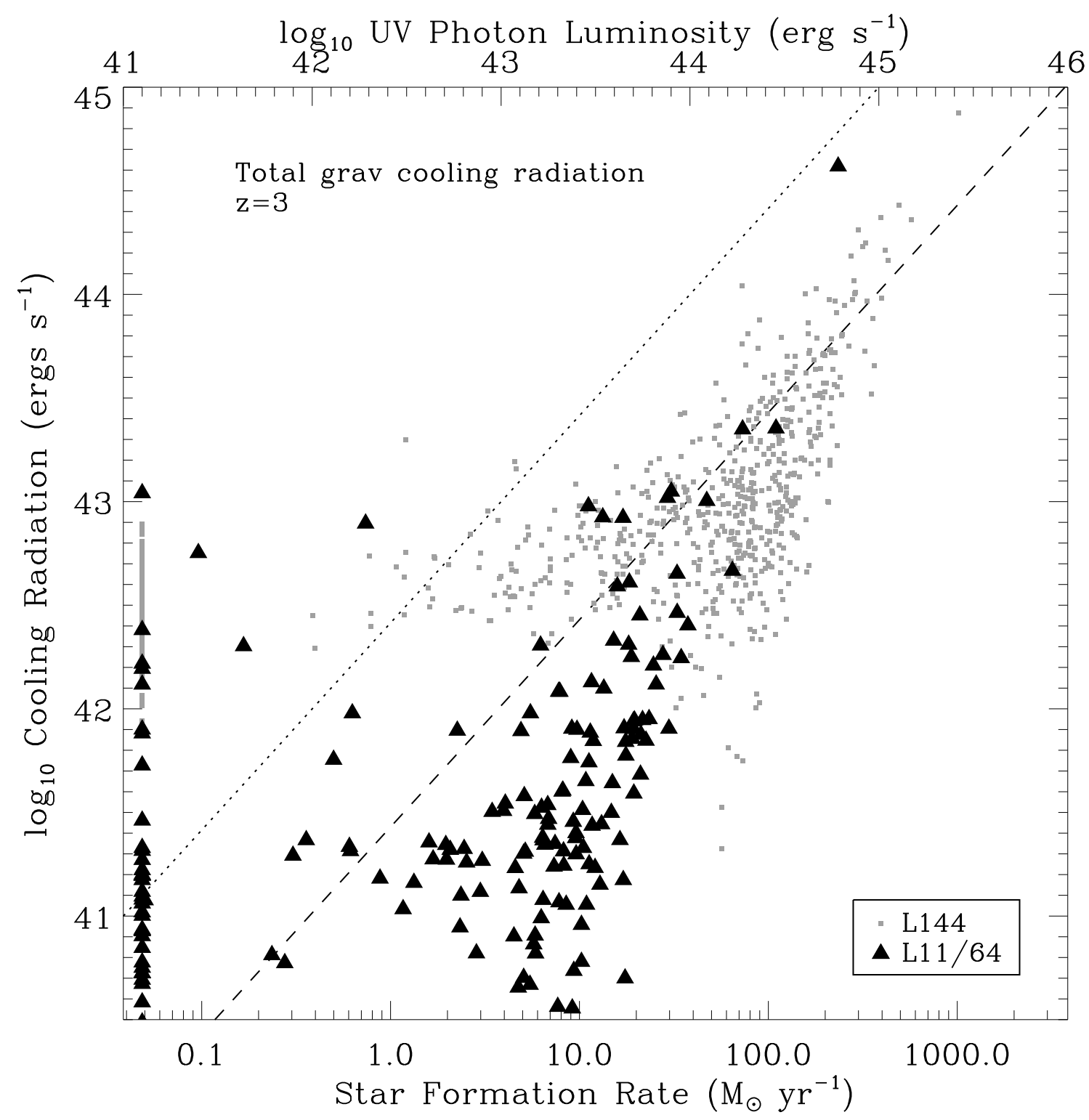

Fig. 7.- The total gravitational cooling radiation as a function of the star formation rate, at $z=3$. The galaxies in the L144 simulation and the L11/64 simulation are plotted as squares and triangles respectively. The Lyman continuum luminosity resulting from the star formation is shown along the top axis. The supernova heating resulting from star formation is shown by the dashed line; galaxies lying on this line have equal gravitational and supernova contributions to the cooling radiation, and gravitational cooling dominates for points above the line. Galaxies on the dotted line would have gravitational cooling radiation equal to the ionizing radiation output of their young stars. Galaxies with no star formation are plotted at the extreme left. 


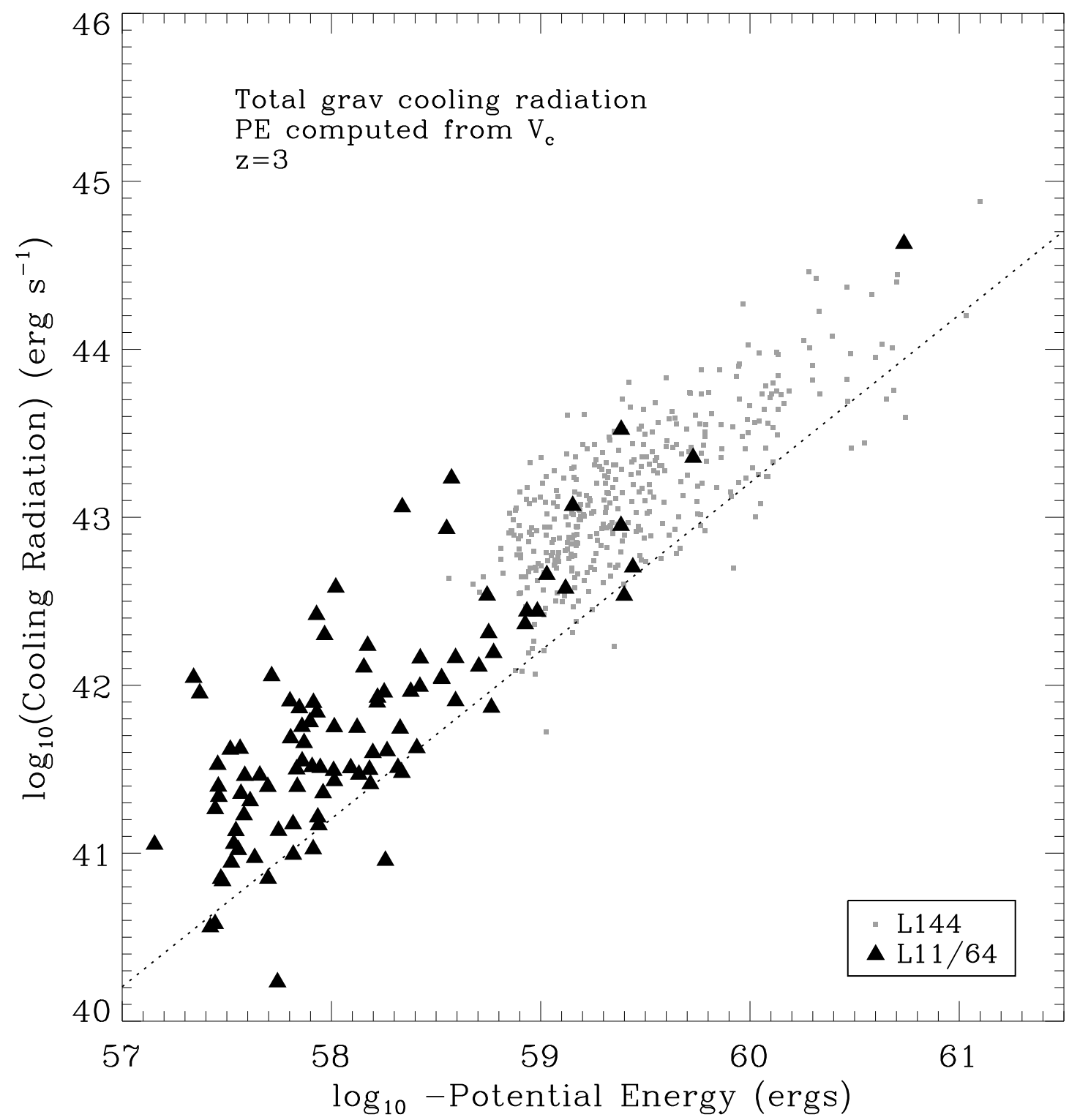

Fig. 8. - Total gravitational cooling vs. potential energy, at $z=3$. The galaxies in the L144 simulation and the L11/64 simulation are plotted as squares and triangles respectively. The potential energy here is simply calculated from $U=M_{b} V_{\text {circ }}^{2}$, where $V_{\text {circ }}$ is the measured circular velocity and $M_{b}$ is the baryonic mass. The dotted line shows the potential energy divided by the cosmic time. 


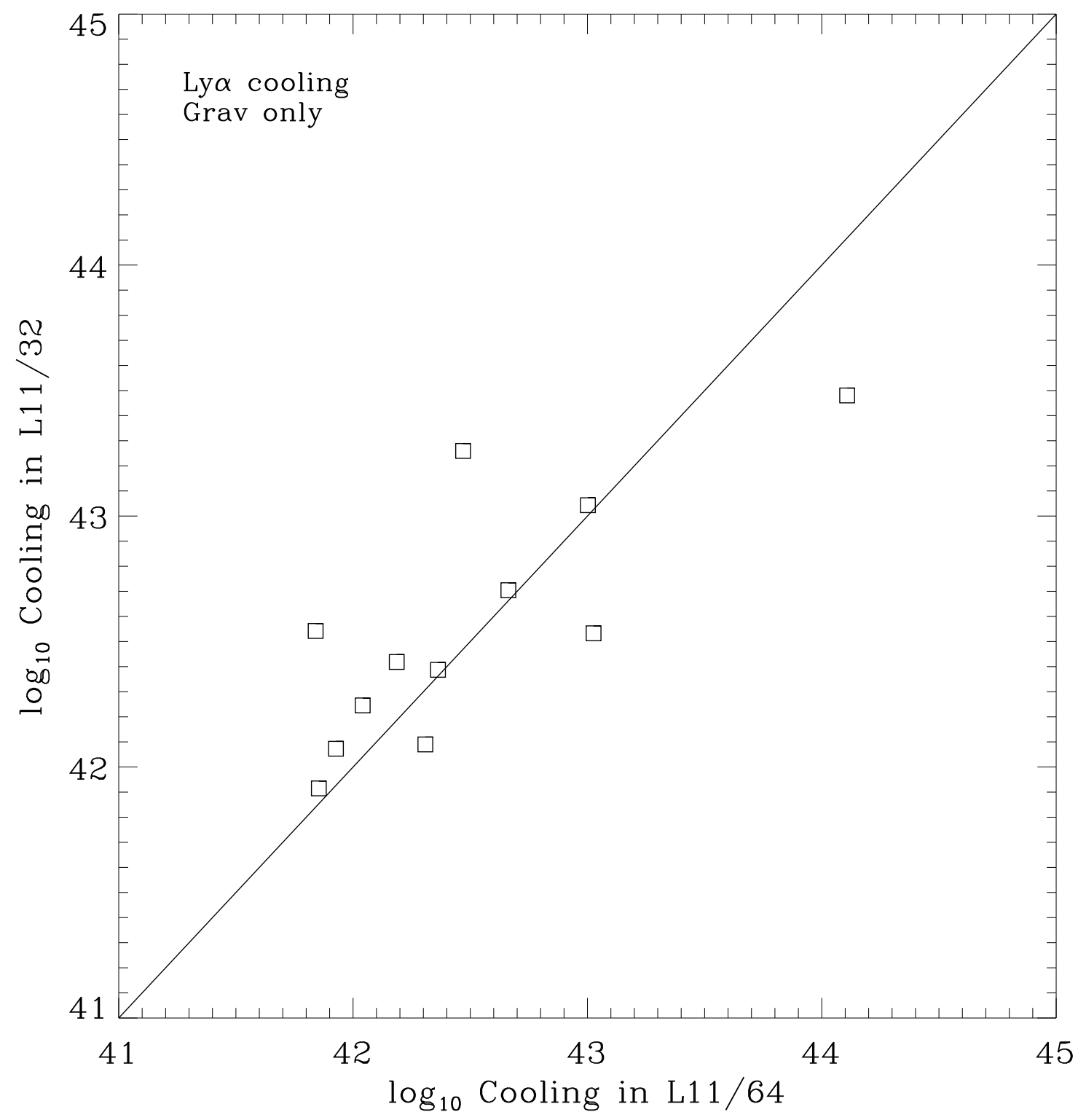

Fig. 9. - Cooling luminosity in Ly $\alpha$ at two resolutions at $z=3$. Galaxies from the lower resolution L11/32 simulation, which has the same resolution as L144, are plotted versus the same galaxies from the higher resolution, L11/64 simulation. 